Article

\title{
Agronomic and Metabolomic Side-Effects of a Divergent Selection for Indol-3-Ylmethylglucosinolate Content in Kale (Brassica oleracea var. acephala)
}

\author{
Jorge Poveda $^{1}$, Pablo Velasco ${ }^{2}\left(\right.$, Antonio de Haro ${ }^{3} \oplus$, Tor J. Johansen ${ }^{4}$, Alex C. McAlvay ${ }^{5} \oplus$, Christian Möllers ${ }^{6}$, \\ Jørgen A.B. Mølmann ${ }^{4} \mathbb{D}$, Elena Ordiales ${ }^{7}$ and Víctor M. Rodríguez ${ }^{2, *} \mathbb{C}$ \\ 1 Institute of Agrobiotechnology, Public University of Navarre, 31006 Pamplona, Spain; \\ jorge.poveda@unavarra.es \\ 2 Mision Biologica de Galicia (MBG-CSIC), 36143 Pontevedra, Spain; pvelasco@mbg.csic.es \\ 3 Institute of Sustainable Agriculture (CSIC), 14004 Córdoba, Spain; adeharobailon@ias.csic.es \\ 4 Norwegian Institute of Bioeconomy Research (NIBIO), P.O. Box 115, NO-1431 Ås, Norway; \\ tor.johansen@nibio.no (T.J.J.); jorgen.molmann@nibio.no (J.A.B.M.) \\ 5 Institute of Economic Botany, The New York Botanical Garden, New York, NY 10458, USA; \\ amcalvay@nybg.org \\ updates \\ Citation: Poveda, J.; Velasco, P.; \\ de Haro, A.; Johansen, T.J.; \\ McAlvay, A.C.; Möllers, C.; \\ 6 Department of Crop Science, Georg-August-Universität Göttingen, 37075 Göttingen, Germany; \\ cmoelle2@gwdg.de \\ 7 Centro Tecnológico Nacional Agroalimentario, 06195 Badajoz, Spain; eordiales@ctaex.com \\ * Correspondence: vmrodriguez@mbg.csic.es; Tel.: +34-986-85-4800
} Mølmann, J.A.B.; Ordiales, E.; Rodríguez, V.M. Agronomic and Metabolomic Side-Effects of a Divergent Selection for Indol-3-Ylmethylglucosinolate Content in Kale

(Brassica oleracea var. acephala). Metabolites 2021, 11, 384. https://doi.org/10.3390/ metabo11060384

Academic Editors: Ian Dubery, Lizelle A. Piater and Fidele Tugizimana

Received: 31 March 2021

Accepted: 10 June 2021

Published: 14 June 2021

Publisher's Note: MDPI stays neutral with regard to jurisdictional claims in published maps and institutional affiliations.

Copyright: (c) 2021 by the authors. Licensee MDPI, Basel, Switzerland. This article is an open access article distributed under the terms and conditions of the Creative Commons Attribution (CC BY) license (https:// creativecommons.org/licenses/by/ $4.0 /)$.

\begin{abstract}
Brassica oleracea var. acephala (kale) is a cruciferous vegetable widely cultivated for its leaves and flower buds in Europe and a food of global interest as a "superfood". Brassica crops accumulate phytochemicals called glucosinolates (GSLs) which play an important role in plant defense against biotic stresses. Studies carried out to date suggest that GSLs may have a role in the adaptation of plants to different environments, but direct evidence is lacking. We grew two kale populations divergently selected for high and low indol-3-ylmethylGSL (IM) content (H-IM and L-IM, respectively) in different environments and analyzed agronomic parameters, GSL profiles and metabolomic profile. We found a significant increase in fresh and dry foliar weight in H-IM kale populations compared to L-IM in addition to a greater accumulation of total GSLs, indole GSLs and, specifically, IM and 1-methoxyindol-3-ylmethylGSL (1MeOIM). Metabolomic analysis revealed a significant different concentration of 44 metabolites in $\mathrm{H}$-IM kale populations compared to L-IM. According to tentative peak identification from MS interpretation, $80 \%$ were phenolics, including flavonoids (kaempferol, quercetin and anthocyanin derivates, including acyl flavonoids), chlorogenic acids (esters of hydroxycinnamic acids and quinic acid), hydroxycinnamic acids (ferulic acid and $p$-coumaric acid) and coumarins. H-IM kale populations could be more tolerant to diverse environmental conditions, possibly due to GSLs and the associated metabolites with predicted antioxidant potential.
\end{abstract}

Keywords: glucosinolates; kale; abiotic stress; phenolics; flavonoids

\section{Introduction}

Crops belonging to the genus Brassica are among the top ten most agronomically and economically important vegetable species in the world. These crops show high morphological and agronomic diversity and are cultivated mainly in temperate regions of the Northern Hemisphere [1]. Kale (Brassica oleracea var. acephala) is a leafy vegetable crop that is becoming popular as a "superfood", due to its nutritional value (rich in $\mathrm{Ca}^{2+}$, folate, riboflavin, vitamins $\mathrm{C}, \mathrm{K}$ and $\mathrm{A}$ ), phytochemical composition (including polyphenols, glucosinolates, terpenoids and carotenoids), and its high anticarcinogenic and antioxidant potential [2]. Among Brassica phytochemicals, glucosinolates (GSLs)-sulfur compounds 
derived from amino acids [3] — are the most well-studied compounds. A primary function of GSLs in plants includes defense against pathogens and pests $[4,5]$. Inside of cells GSLs are chemically stable but, upon cell disruption due to tissue damage, GSLs are exposed to the activity of myrosinase and associated proteins, resulting in glucosinolate hydrolysis products (GHPs) [3]. There are numerous examples of the GHPs antimicrobial activity against plant pathogens, through different mechanisms such as affecting metabolic pathways or cellular structures [6,7]. Against herbivores, mainly insects, the mechanism involved in the defensive capacity of GHPs is direct toxicity by ingestion [8]. However, it has been described how GSLs can also play a role as attractants of beneficial insects, such as pollinators [9] or parasitoids [10], as well as insect-pest of Brassica crops [11].

Although GSLs are well-studied in their fundamental role in biotic interactions, there is a lack of understanding of the role of these compounds in other physiological processes. Some evidence indicates that GSLs play a key role as signaling compounds for flowering time, stomatal closure or water transport and may affect auxin signaling [12-14]. These alternative roles suggest that GSLs may play a role in adaptation of GSL-containing plants to different environments. Consistent with this possibility, several studies report that abiotic stress conditions such as drought, salinity and extreme temperatures, can impact GSL accumulation in Brassica plants (for review see [15]). Bonasia et al. [16] also show that in wild rocket (Diplotaxis tenuifolia), GSL content is affected by growing seasons, being higher when plants are cultivated during a winter-spring season. Interestingly, plants with higher GSL content also show a higher yield. In kale, it was reported that exposure to different temperatures significantly modifies the GSL profiles of plant tissues [17]. These findings open a question of whether GSLs may play a role on plant adaptation to different environments.

Different strategies can be used to elucidate the biological effects of GSLs in plants. The most widespread include the use of Arabidopsis thaliana mutants [4,18], comparison between species or ecotypes with different GSL profiles [19,20], or the use of populations obtained by divergent selection. Our research group previously carried out three divergent selection programs targeting the three primary GSL compounds in kale. We bred a local Spanish landrace population, MBG-BRS0062, for three cycles and obtained six divergent populations: high and low 3-(methylsulfinyl)propylGSL ( $3 \mathrm{mSOp}$ ) content, high and low 2-propenylGSL content and high and low indolylmethylGSL (IM) content [21]. Populations high in IM (H-IM) and low in IM (L-IM) were used in the present study. The differential IM accumulation of these individuals is related to variations in the expression of the CYP81F2 gene in kale tissues [21]. These populations have previously been used to suggest the role of GSL in the reduction of larval weight in different lepidopteran and aphid pests (Mamestra brassicae, Pieris rapae and Brevicoryne brassicae) [22,23].

In a previous study, no significant differences were reported in the agronomic parameters and profile of GSLs between the kale populations H-IM and L-IM [21]. We decided to cultivate both populations in varied environments to determine how divergent selection for IM content in kale affects agronomic traits evaluated in different environments. Since, it is plausible that a higher accumulation of IM after three cycles of divergent selection may also produce a reorganization of the plant metabolome, we also performed an un-targeted metabolomics analysis to study the role of these changes on plant adaptability.

\section{Results}

\subsection{Differences in Agronomic Parameters}

The analysis of variance for plant height showed differences only between locations (L), but not between populations $(G)$ or the $L \times G$ interaction. However, there were significant differences between fresh and dry weight between H-IM and L-IM ( $p=0.0122$ and 0.0213, respectively). The mean fresh and dry weights were significantly higher in H-IM (1563 g and $172 \mathrm{~g}$, respectively) than in L-IM (1342 $\mathrm{g}$ and $145 \mathrm{~g}$, respectively). The interaction between populations and locations was significant only for fresh weight, but fresh weight was higher in H-IM than L-IM in any location (Figure 1). 
(a)

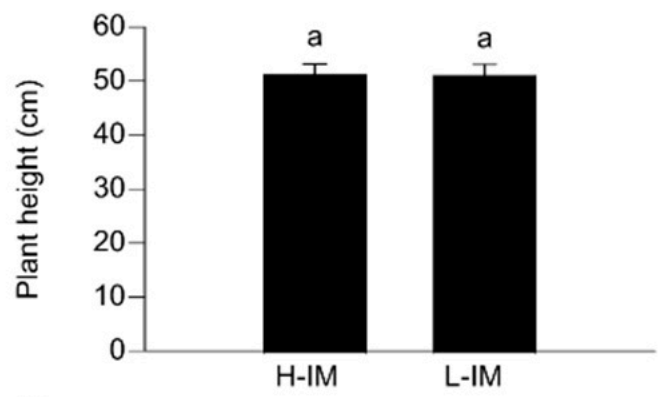

(b)



(c)

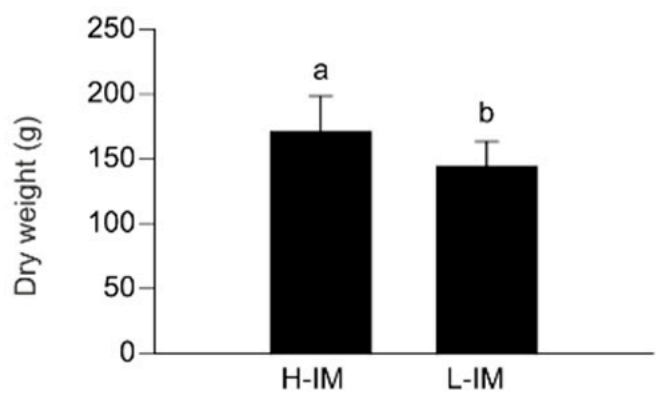

Figure 1. Agronomic parameters in two populations of kale from divergently selected populations with varied indol-3-ylmethylGSL (IM) content (H-IM: High IM content; L-IM: Low IM content). (a) Means of plant height of kale populations in all locations. (b) Means of fresh weight of 25 leaves of kale populations in all locations. (c) Means of dry weight of 25 leaves of kale populations in all locations. Error bars represent \pm standard deviation (SD). Within each panel, different letters indicate significant differences between populations (ANOVA, $p$-value $\leq 0.05$ ). Complete ANOVA table results is presented as Supplementary Material (Table S1).

\subsection{GSL Profiles}

Our results indicated that the IM content was significantly higher in the H-IM population than in the L-IM population (Figure 2). The analysis of total GSL content of the divergent populations indicated significantly larger amounts in the H-IM $(34.1 \mu \mathrm{mol} / \mathrm{g}$ dry weight) population than in the L-IM population $(24.3 \mu \mathrm{mol} / \mathrm{g}$ dry weight $)(p<0.01)$. This higher levels of GSLs are mainly due to the accumulation of indole GSLs $(p<0.01)$ in the H-IM population $(21.4 \mu \mathrm{mol} / \mathrm{g}$ dry weight) since we did not observe differences between both populations for total aliphatic GSLs content ( $p=0.0989)$. Aside from IM, we only observed a significant increase on the accumulation of 1MeOIM $(p<0.01)$ in the $\mathrm{H}$-IM population $(6.6 \mu \mathrm{mol} / \mathrm{g}$ dry weight $)$ and a decrease on the accumulation of $3 \mathrm{mSOp}$ $(6.4 \mu \mathrm{mol} / \mathrm{g}$ dry weight) $(p=0.0008)$ (Figure 2$)$, indicating that the selection method was quite specific in increasing IM content. 


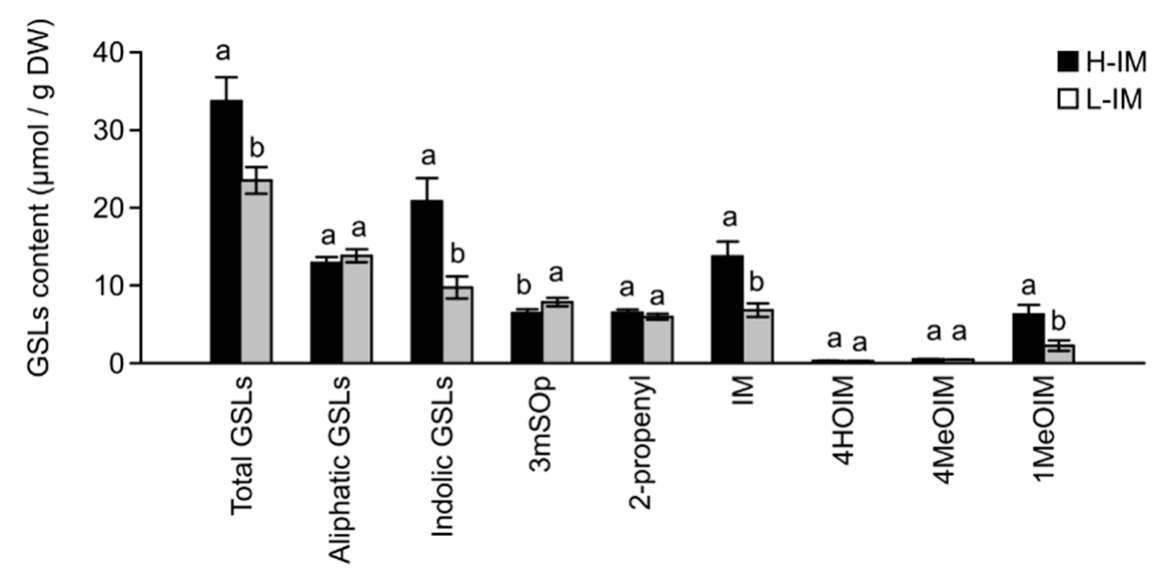

Figure 2. Glucosinolates content in two populations of kale from divergently selected populations with varied indol-3-ylmethylGSL (IM) content (H-IM: High IM content; L-IM: Low IM content). Error bars represent \pm standard error (SE). Within each glucosinolate category different letters indicate significant differences between divergent populations (ANOVA, $p$-value $\leq 0.05$ ). Complete ANOVA table results is presented as Supplementary Material (Table S2). Abbreviations: GSLs: Glucosinolates, 3mSOp: 3-(methylsulfinyl)propylGSLs, 2-propenyl: 2-propenylGSL, IM: indol-3ylmethylGSL, 4HOIM: 4-hydroxyindol-3-ylmethylGSL, 4MeOIM: 4-methoxyindol-3-ylmethylGSL and 1MeOIM: 1-methoxyindol-3-ylmethylGSL.

\subsection{Metabolomic Profiles}

In order to identify the metabolomic changes that could take place in the kale population due to divergent selection on IM content, we performed a non-targeted metabolomics analysis. Statistical univariate analyses reported 109 features that were differentially accumulated in the H-IM and L-IM populations (Figure 3). Data was then hand-filtered, taking into account retention time and correlation coefficients to remove features that were most likely due to in-source fragmentation of metabolites. Ultimately, 67 features of these were considered to be true metabolites ( 30 detected in negative and 37 in positive ionization modes) (Table 1). Forty-four of these metabolites had increased concentration in H-IM populations compared to L-IM populations, while 23 metabolites had decreased concentrations. IM was not among the selected metabolites since though showed significantly higher levels in the H-IM population vs. L-IM ( $p$ value $=0.001$ ) in the metabolomics analysis, did not fit the fold change condition.

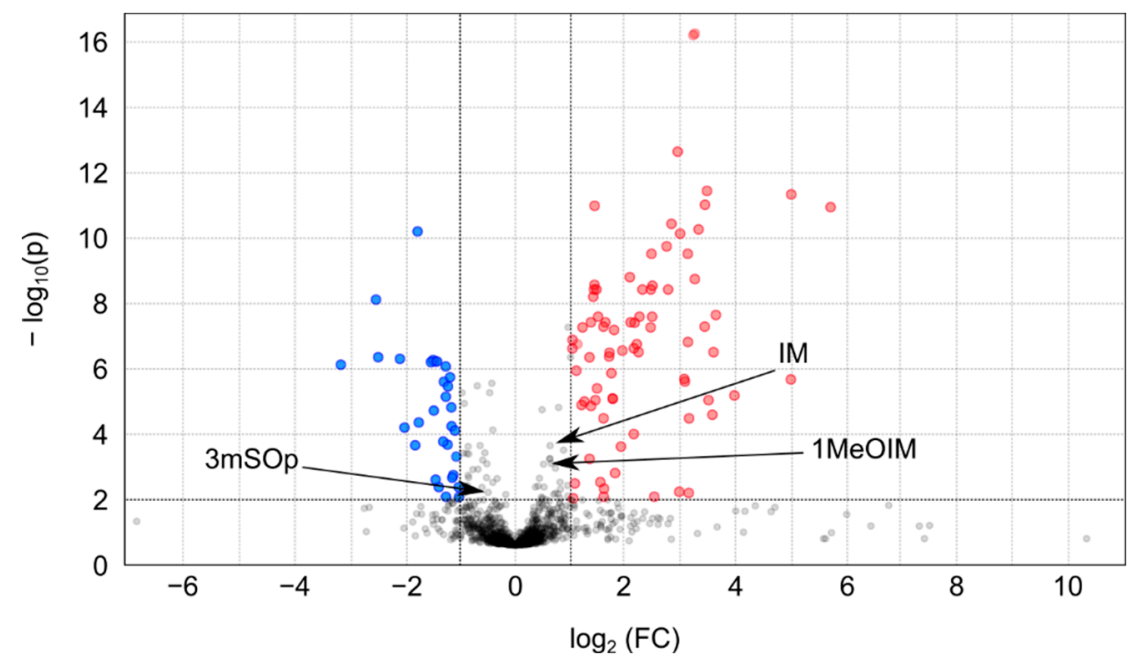

Figure 3. Volcano plot representing the detected features in the non-targeted metabolomic analysis. The $y$-axis represents the negative decade logarithm of the significance value (FDR) and the x-axis 
represents the $\log _{2}$ of fold change (H-IM vs. L-IM). Levels of features with a $-\log _{10}(p) \leq 1.3$ and a $\left|\log _{2}(F C)\right| \geq 1$ are considered to be differentially accumulated in both populations. Significantly up-regulated features are represented by red circles and down-regulated features are represented by blue circles. Grey circles represent insignificant features. Positions of significant GSLs in the target analysis are represented by arrows (3mSOp: 3-(methylsulfinyl)propylGSL $\left(\log _{2}(\mathrm{FC})=-0.39 ;-\log _{10}(\mathrm{p})=1.91\right) ; \mathrm{IM}$ : indol-3-ylmethylGSL $\left(\log _{2}(\mathrm{FC})=0.36 ;-\log _{10}(\mathrm{p})=2.72\right)$; 1MeOIM: 1-methoxyindol-3-ylmethylGSL $\left.\left(\log _{2}(\mathrm{FC})=0.54 ;-\log _{10}(\mathrm{p})=1.55\right)\right)$.

Table 1. Tentative identification of metabolites with a $\left|\log _{2}(F C)\right| \geq 1$ and statistically significant difference (FDR $\left.\leq 0.05\right)$ between populations (High-Indol-3-ylmethylGSL vs. Low-Indol-3-ylmethylGSL content). Metabolites are sorted by ionization mode. References to the identification of compounds in the Brassicaceae plant family is included when available. Compounds in bold: fragmentation spectra match with authentic standard. Underlined compounds: retention time match with authentic standard.

\begin{tabular}{|c|c|c|c|c|c|c|c|c|c|}
\hline$m / z$ & $\begin{array}{l}\text { Neutral } \\
\text { Mass }\end{array}$ & Ionization & $\begin{array}{c}\text { RT } \\
\text { (Min) }\end{array}$ & $\log _{2}(\mathrm{FC})$ & $\begin{array}{l}\text { Molecular } \\
\text { Formula }\end{array}$ & $\begin{array}{l}\text { Theoretical } \\
\text { Mass }\end{array}$ & $\begin{array}{c}\text { Mass } \\
\text { Deviation } \\
(\mathrm{ppm})\end{array}$ & Fragments & Tentative Identification \\
\hline \multicolumn{10}{|c|}{ A. Peaks detected in negative ionization mode } \\
\hline 337.0937 & 338.1010 & {$[\mathrm{M}-\mathrm{H}]^{-}$} & 10.1 & 2.1 & $\mathrm{C}_{16} \mathrm{H}_{18} \mathrm{O}_{8}$ & 338.1002 & 2.514 & $119.05,163.04,191.06$ & $\begin{array}{l}\text { coumaroylquinic acid } \\
\text { isomer } 1 \text { [24] }\end{array}$ \\
\hline 771.1991 & 772.2064 & {$[\mathrm{M}-\mathrm{H}]^{-}$} & 10.3 & -1.1 & $\mathrm{C}_{33} \mathrm{H}_{40} \mathrm{O}_{21}$ & 772.2062 & 0.176 & $\begin{array}{c}283.04,609.17,255.03 \\
422.07,446.10\end{array}$ & $\begin{array}{l}\text { kaempferol } \\
\text { sophoroside-hexoside [25] }\end{array}$ \\
\hline 675.1938 & 338.1005 & {$[2 \mathrm{M}-\mathrm{H}]^{-}$} & 10.4 & 2.2 & $\mathrm{C}_{16} \mathrm{H}_{18} \mathrm{O}_{8}$ & 338.1002 & 1.035 & $163.04,337.09,191.06$ & $\begin{array}{l}\text { coumaroylquinic acid } \\
\text { isomer } 2 \text { [24] }\end{array}$ \\
\hline 325.0937 & 326.1010 & {$[\mathrm{M}-\mathrm{H}]^{-}$} & 10.8 & 1.8 & $\mathrm{C}_{15} \mathrm{H}_{18} \mathrm{O}_{8}$ & 326.1007 & 0.889 & $119.05,163.04$ & coumaroylglucoside [26] \\
\hline 489.1252 & 980.2650 & {$[\mathrm{M}-2 \mathrm{H}]^{-2}$} & 11.2 & -1.2 & $\mathrm{C}_{40} \mathrm{H}_{52} \mathrm{O}_{28}$ & 980.2645 & 0.490 & $\begin{array}{c}609.15,284.03,933.25 \\
101.02\end{array}$ & $\begin{array}{l}\text { kaempferol sophoroside- } \\
\text { (dihydroxymethoxy) } \\
\text { sophoroside }^{1}\end{array}$ \\
\hline 628.1641 & 629.1714 & {$[\mathrm{M}-\mathrm{H}]^{-}$} & 11.9 & -1.0 & & & & $466.11,284.03,161.02$ & $\begin{array}{l}\text { kaempferol hexoside } \\
\text { derivative or isomer }\end{array}$ \\
\hline 625.1416 & 626.1488 & {$[\mathrm{M}-\mathrm{H}]^{-}$} & 11.9 & 3.1 & $\mathrm{C}_{27} \mathrm{H}_{30} \mathrm{O}_{17}$ & 626.1483 & 0.854 & $299.03,271.04$ & quercetin sophoroside [27] \\
\hline 337.0932 & 338.1005 & {$[\mathrm{M}-\mathrm{H}]^{-}$} & 12.0 & 1.5 & $\mathrm{C}_{16} \mathrm{H}_{18} \mathrm{O}_{8}$ & 338.1002 & 0.917 & $\begin{array}{c}173.05,119.05,93.03 \\
163.04\end{array}$ & $\begin{array}{l}\text { coumaroylquinic acid } \\
\text { isomer } 3 \text { [24] } \\
\text { kaempferol }\end{array}$ \\
\hline 569.1518 & 1140.3181 & {$[\mathrm{M}-2 \mathrm{H}]^{-2}$} & 12.6 & -1.8 & $\mathrm{C}_{50} \mathrm{H}_{60} \mathrm{O}_{30}$ & 1140.3170 & 0.991 & $\begin{array}{c}488.12,407.09,815.20 \\
205.05,284.03\end{array}$ & $\begin{array}{l}\text { sinapoylsophoroside- } \\
\text { gentobioside } \\
{[28]}\end{array}$ \\
\hline 635.1728 & 1272.3601 & {$[\mathrm{M}-2 \mathrm{H}]^{-2}$} & 12.7 & -1.3 & $\mathrm{C}_{55} \mathrm{H}_{68} \mathrm{O}_{34}$ & 1272.3592 & 0.684 & $\begin{array}{c}\text { 473.12, } 635.17,947.24, \\
119.04,161.05,263.07 \\
323.10\end{array}$ & $\begin{array}{l}\text { kaempferol } \\
\text { feruloylpentaglucoside } \\
{\left[\begin{array}{l}29]\end{array}\right.}\end{array}$ \\
\hline 609.1463 & 610.1536 & {$[\mathrm{M}-\mathrm{H}]^{-}$} & 12.8 & 2.1 & $\mathrm{C}_{27} \mathrm{H}_{30} \mathrm{O}_{16}$ & 610.1534 & 0.295 & $477.09,285.04$ & $\begin{array}{l}\text { kaempferol dihexoside } \\
\text { [30] }\end{array}$ \\
\hline 554.1465 & 1110.3075 & {$[\mathrm{M}-2 \mathrm{H}]^{-2}$} & 12.8 & -1.3 & $\mathrm{C}_{49} \mathrm{H}_{58} \mathrm{O}_{29}$ & 1110.3064 & 1.013 & $\begin{array}{l}473.12,392.09,785.19, \\
284.03,175.04,609.14\end{array}$ & $\begin{array}{l}\text { kaempferol } \\
\text { feruloylsophoroside- } \\
\text { cellobioside } \\
{[28]}\end{array}$ \\
\hline 337.0935 & 338.1008 & {$[\mathrm{M}-\mathrm{H}]^{-}$} & 12.9 & 1.1 & $\mathrm{C}_{16} \mathrm{H}_{18} \mathrm{O}_{8}$ & 338.1002 & 1.863 & $\begin{array}{c}173.04,93.03,119.05 \\
163.04,111.04\end{array}$ & $\begin{array}{l}\text { coumaroylquinic acid } \\
\text { isomer } 4 \text { [24] }\end{array}$ \\
\hline 755.2047 & 756.2119 & {$[\mathrm{M}-\mathrm{H}]^{-}$} & 13.2 & 1.8 & $\mathrm{C}_{33} \mathrm{H}_{40} \mathrm{O}_{20}$ & 756.2113 & 0.847 & $\begin{array}{c}283.04,255.04,609.17 \\
227.05,430.10\end{array}$ & $\begin{array}{c}\text { kaempferol } \\
\text { gentiobioside-rhamnoside } \\
\text { isomer } 1 \text { [31] }\end{array}$ \\
\hline 337.0935 & 338.1014 & {$[\mathrm{M}-\mathrm{H}]^{-}$} & 13.9 & 1.5 & $\mathrm{C}_{16} \mathrm{H}_{18} \mathrm{O}_{8}$ & 338.1002 & 3.668 & 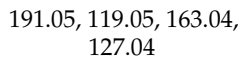 & $\begin{array}{c}\text { coumaroylquinic acid } \\
\text { isomer } 5 \text { [24] } \\
\text { quercetin- }\end{array}$ \\
\hline 771.2003 & 772.2076 & {$[\mathrm{M}-\mathrm{H}]^{-}$} & 14.2 & 3.6 & $\mathrm{C}_{33} \mathrm{H}_{40} \mathrm{O}_{21}$ & 772.2062 & 1.782 & $447.09,625.14,301.03$ & $\begin{array}{c}\text { quercetın- } \\
\text { (rhamnosylhexoside)- } \\
\text { hexoside or } \\
\text { isomer }\end{array}$ \\
\hline 625.142 & 626.1493 & {$[\mathrm{M}-\mathrm{H}]^{-}$} & 14.3 & -1.2 & $\mathrm{C}_{27} \mathrm{H}_{30} \mathrm{O}_{17}$ & 626.1483 & 1.526 & $\begin{array}{c}300.03,179.0,445.08 \\
463.09\end{array}$ & quercetin dihexoside [32] \\
\hline 609.1468 & 610.1540 & {$[\mathrm{M}-\mathrm{H}]^{-}$} & 14.3 & 1.6 & $\mathrm{C}_{27} \mathrm{H}_{30} \mathrm{O}_{16}$ & 610.1534 & 1.048 & $\begin{array}{c}446.08,283.02,463.09 \\
301.03\end{array}$ & $\begin{array}{c}\text { quercetin } \\
\text { hexoside-rhamnoside [33] } \\
\text { kaempferol }\end{array}$ \\
\hline 755.2044 & 756.2117 & {$[\mathrm{M}-\mathrm{H}]^{-}$} & 15.0 & 2.9 & $\mathrm{C}_{33} \mathrm{H}_{40} \mathrm{O}_{20}$ & 756.2113 & 0.517 & $609.15,431.10,285.04$ & $\begin{array}{c}\text { gentiobioside-rhamnoside } \\
\text { isomer } 2 \text { [34] }\end{array}$ \\
\hline 593.1518 & 594.1584 & {$[\mathrm{M}-\mathrm{H}]^{-}$} & 15.2 & 5.0 & $\mathrm{C}_{27} \mathrm{H}_{30} \mathrm{O}_{15}$ & 594.1585 & 0.143 & $430.09,447.09,285.04$ & $\begin{array}{c}\text { kaempferol rutinoside or } \\
\text { isomer }\end{array}$ \\
\hline 639.1569 & 640.1642 & {$[\mathrm{M}-\mathrm{H}]^{-}$} & 15.4 & -3.2 & $\mathrm{C}_{28} \mathrm{H}_{32} \mathrm{O}_{17}$ & 640.1640 & 0.305 & $314.04,459.09,609.15$ & (iso)rhamnetin-dihexoside \\
\hline 623.1625 & 624.1705 & {$[\mathrm{M}-\mathrm{H}]^{-}$} & 15.5 & 3.6 & $\mathrm{C}_{28} \mathrm{H}_{32} \mathrm{O}_{16}$ & 624.1690 & 2.339 & $461.11,477.10,315.05$ & $\begin{array}{l}\text { (iso)rhamnetin- } \\
\text { rhamnosylhexoside }\end{array}$ \\
\hline 657.1752 & 1316.3649 & {$[\mathrm{M}-2 \mathrm{H}]^{-2}$} & 17.0 & -1.3 & $\mathrm{C}_{60} \mathrm{H}_{68} \mathrm{O}_{33}$ & 1316.3643 & 0.437 & $\begin{array}{c}576.14,284.03,175.04, \\
205.05\end{array}$ & $\begin{array}{c}\text { kaempferol- } \\
\text { (feruloyl)(sinapoyl)- } \\
\text { trihexoside-hexoside or } \\
\text { isomer } 1\end{array}$ \\
\hline
\end{tabular}


Table 1. Cont.

\begin{tabular}{|c|c|c|c|c|c|c|c|c|c|}
\hline$m / z$ & $\begin{array}{l}\text { Neutral } \\
\text { Mass }\end{array}$ & Ionization & $\begin{array}{l}\text { RT } \\
\text { (Min) }\end{array}$ & $\log _{2}(\mathrm{FC})$ & $\begin{array}{l}\text { Molecular } \\
\text { Formula }\end{array}$ & $\begin{array}{l}\text { Theoretical } \\
\text { Mass }\end{array}$ & $\begin{array}{c}\text { Mass } \\
\text { Deviation } \\
(\mathrm{ppm})\end{array}$ & Fragments & Tentative Identification \\
\hline 709.1998 & 710.2071 & {$[\mathrm{M}-\mathrm{H}]^{-}$} & 17.1 & 1.6 & $\mathrm{C}_{32} \mathrm{H}_{38} \mathrm{O}_{18}$ & 710.2058 & 1.816 & $\begin{array}{c}485.13,161.03,223.06 \\
179.04\end{array}$ & $\begin{array}{c}\text { kaempferol derivative or } \\
\text { isomer }\end{array}$ \\
\hline 657.1745 & 1316.3641 & {$[\mathrm{M}-2 \mathrm{H}]^{-2}$} & 17.2 & -1.5 & $\mathrm{C}_{60} \mathrm{H}_{68} \mathrm{O}_{33}$ & 1316.3643 & 0.141 & $\begin{array}{c}576.15,284.03,205.05 \\
175.04,947.26\end{array}$ & $\begin{array}{c}\text { kaempferol- } \\
\text { (feruloyl)(sinapoyl)- } \\
\text { trihexoside-hexoside or } \\
\text { isomer } 2\end{array}$ \\
\hline 415.1977 & 416.2050 & {$[\mathrm{M}-\mathrm{H}]^{-}$} & 17.6 & 1.8 & $\mathrm{C}_{20} \mathrm{H}_{32} \mathrm{O}_{9}$ & 416.2046 & 0.781 & $\begin{array}{c}44.99, \text {, } 71.02,113.03, \\
101.02\end{array}$ & $\begin{array}{c}\text { nicotinic acid hexoside } \\
\text { derivative }\end{array}$ \\
\hline 709.4687 & 710.4760 & {$[\mathrm{M}-\mathrm{H}]^{-}$} & 28.7 & -1.8 & & & & & \\
\hline 683.4655 & 684.4728 & {$[\mathrm{M}-\mathrm{H}]^{-}$} & 29.5 & -1.8 & & & & & \\
\hline 683.4659 & 684.4732 & {$[\mathrm{M}-\mathrm{H}]^{-}$} & 29.8 & -2.1 & & & & & \\
\hline 709.4807 & 710.4880 & {$[\mathrm{M}-\mathrm{H}]^{-}$} & 29.8 & -2.0 & & & & & \\
\hline \multicolumn{10}{|c|}{ B. Peaks detected in positive ionization mode } \\
\hline 110.0702 & 109.0629 & {$[\mathrm{M}+\mathrm{H}]^{+}$} & 0.8 & 1.1 & & & & 47.77 & \multirow{7}{*}{$\begin{array}{c}\text { kynurenic acid } \\
\text { feruloyl derivative } \\
\text { coumarin isomer } \mathbf{1}^{2} \\
\text { cyanidin-hexoside or } \\
\text { isomer } \\
\text { coumaroylquinic acid } \\
\text { isomer 2 [24] } \\
\text { feruloyl derivative }\end{array}$} \\
\hline 190.0502 & 189.0431 & {$[\mathrm{M}+\mathrm{H}]^{+}$} & 9.7 & -1.5 & $\mathrm{C}_{10} \mathrm{H}_{7} \mathrm{NO}_{3}$ & 189.0426 & 2.412 & $\begin{array}{c}116.05,162.05,89.04 \\
144.04\end{array}$ & \\
\hline 361.0893 & 360.0820 & {$[\mathrm{M}+\mathrm{H}]^{+}$} & 10.1 & 1.1 & & & & $147.05,167.06,91.05$ & \\
\hline 147.0433 & 146.0360 & {$[\mathrm{M}+\mathrm{H}]^{+}$} & 10.1 & 1.4 & $\mathrm{C}_{9} \mathrm{H}_{6} \mathrm{O}_{2}$ & 146.0368 & 5.478 & $65.04,91.05$ & \\
\hline 449.1079 & 448.1006 & {$[\mathrm{M}+\mathrm{H}]^{+}$} & 10.2 & -1.2 & $\mathrm{C}_{21} \mathrm{H}_{20} \mathrm{O}_{11}$ & 448.1006 & 0.036 & $305.07,287.05$ & \\
\hline 339.1074 & 338.1001 & {$[\mathrm{M}+\mathrm{H}]^{+}$} & 10.4 & 1.2 & $\mathrm{C}_{16} \mathrm{H}_{18} \mathrm{O}_{8}$ & 338.1002 & 0.177 & $147.04,119.05,91.05$ & \\
\hline 361.0894 & 360.0821 & {$[\mathrm{M}+\mathrm{H}]^{+}$} & 10.4 & 1.1 & & & & $\begin{array}{c}147.04,167.05,140.99 \\
91.05\end{array}$ & \\
\hline 803.2232 & 802.2160 & {$[\mathrm{M}+\mathrm{H}]^{+}$} & 11.4 & -2.5 & $\mathrm{C}_{34} \mathrm{H}_{42} \mathrm{O}_{22}$ & 802.2168 & 1.022 & $317.06,479.12$ & $\begin{array}{l}\text { ophioglonol-dihexoside- } \\
\text { hexoside or } \\
\text { isomer } 1\end{array}$ \\
\hline 147.0429 & 146.0356 & {$[\mathrm{M}+\mathrm{H}]^{+}$} & 12.1 & 1.9 & $\mathrm{C}_{9} \mathrm{H}_{6} \mathrm{O}_{2}$ & 146.0368 & 8.217 & $\begin{array}{c}65.04,91.05,63.03 \\
55.05\end{array}$ & \multirow{6}{*}{$\begin{array}{c}{\text { coumarin isomer } 2^{2}}{ }^{\text {cyanidin }} \\
\text { sophoroside-hexoside [35] } \\
\text { coumarin isomer } 3^{2} \\
\text { ophioglonol-dihexoside- } \\
\text { hexoside or } \\
\text { isomer } 2 \\
\text { cyanidin- } \\
\text { rhamnosylhexoside- } \\
\text { hexoside or } \\
\text { isomer 1 } \\
\text { cyanidin- } \\
\text { (feruloyldihexoside)- } \\
\text { hexoside or } \\
\text { isomer }\end{array}$} \\
\hline 773.2121 & 772.2049 & {$[\mathrm{M}+\mathrm{H}]^{+}$} & 12.6 & 1.5 & $\mathrm{C}_{33} \mathrm{H}_{41} \mathrm{O}_{21}^{+}$ & 772.2062 & 1.740 & $287.05,303.05,449.10$ & \\
\hline 147.0440 & 146.0367 & {$[\mathrm{M}+\mathrm{H}]^{+}$} & 12.9 & 1.7 & $\mathrm{C}_{9} \mathrm{H}_{6} \mathrm{O}_{2}$ & 146.0368 & 0.342 & $91.05,65.04,53.04$ & \\
\hline 803.2232 & 802.2160 & {$[\mathrm{M}+\mathrm{H}]^{+}$} & 13.1 & -2.5 & $\mathrm{C}_{34} \mathrm{H}_{42} \mathrm{O}_{22}$ & 802.2168 & 1.022 & $\begin{array}{l}317.06,85.03,145.05 \\
479.12\end{array}$ & \\
\hline 757.2173 & 756.2100 & {$\left[\mathrm{M}+\mathrm{H}^{]+}\right.$} & 13.2 & 2.5 & $\mathrm{C}_{33} \mathrm{H}_{40} \mathrm{O}_{20}$ & 756.2113 & 1.744 & $\begin{array}{c}287.05,85.03,433.11 \\
145.05\end{array}$ & \\
\hline 949.2599 & 948.2527 & {$[\mathrm{M}+\mathrm{H}]^{+}$} & 13.4 & 2.8 & $\mathrm{C}_{43} \mathrm{H}_{48} \mathrm{O}_{24}$ & 948.2536 & 0.938 & $\begin{array}{c}177.05,287.05,339.10 \\
449.10\end{array}$ & \\
\hline 233.1654 & 232.1581 & {$[\mathrm{M}+\mathrm{H}]^{+}$} & 13.5 & 2.5 & & & & & \multirow[b]{2}{*}{$\begin{array}{c}\text { cyanidin- } \\
\text { (sinapoyldihexoside)- } \\
\text { hexoside or } \\
\text { isomer } \\
\text { cyanidin- } \\
\text { (coumaroyldihexoside)- } \\
\text { hexoside } \\
{[36]}\end{array}$} \\
\hline 919.2493 & 918.2420 & {$[\mathrm{M}+\mathrm{H}]^{+}$} & 13.7 & 3.1 & $\mathrm{C}_{44} \mathrm{H}_{51} \mathrm{O}_{25}^{+}$ & 918.2430 & 1.040 & $\begin{array}{c}163.04,287.05,325.09 \\
307.08\end{array}$ & \\
\hline 147.0393 & 146.0320 & {$[\mathrm{M}+\mathrm{H}]^{+}$} & 13.9 & 1.3 & & & & $\begin{array}{l}91.05,65.04,55.06 \\
53.04\end{array}$ & \multirow{5}{*}{$\begin{array}{c}\text { delphinidin-rutinoside- } \\
\text { hexoside } \\
\text { [36] } \\
\text { cyanidin- } \\
\text { (sinapoylhexoside)- } \\
\text { rhamnosylhexoside or } \\
\text { isomer } \\
\text { delphinidin-hexoside } \\
\text { isomer [37] } \\
\text { cyanidin-dihexoside or } \\
\text { isomer 1 }\end{array}$} \\
\hline 773.2127 & 772.2054 & {$[\mathrm{M}+\mathrm{H}]^{+}$} & 14.1 & 3.6 & $\mathrm{C}_{33} \mathrm{H}_{40} \mathrm{O}_{21}$ & 772.2062 & 1.080 & $\begin{array}{c}303.05,85.03,145.05 \\
287.05,449.11\end{array}$ & \\
\hline 963.2752 & 962.2679 & {$[\mathrm{M}+\mathrm{H}]^{+}$} & 14.2 & 2.5 & $\mathrm{C}_{44} \mathrm{H}_{51} \mathrm{O}_{24}^{+}$ & 962.2692 & 1.319 & $\begin{array}{c}287.05,369.12,433.11 \\
207.07\end{array}$ & \\
\hline 465.1027 & 464.0954 & {$[\mathrm{M}+\mathrm{H}]^{+}$} & 14.3 & -1.1 & $\mathrm{C}_{21} \mathrm{H}_{20} \mathrm{O}_{12}$ & 464.0955 & 0.086 & $303.05,85.03,127.04$ & \\
\hline 611.1600 & 610.1527 & {$[\mathrm{M}+\mathrm{H}]^{+}$} & 14.3 & 2.1 & $\mathrm{C}_{27} \mathrm{H}_{30} \mathrm{O}_{16}$ & 610.1534 & 1.082 & $\begin{array}{c}303.05,287.05,85.03 \\
127.04,449,11\end{array}$ & \\
\hline 633.2012 & 632.1940 & {$[\mathrm{M}+\mathrm{H}]^{+}$} & 14.4 & 2.2 & $\mathrm{C}_{27} \mathrm{H}_{36} \mathrm{O}_{17}$ & 632.1953 & 2.048 & $\begin{array}{c}147.04,165.05,127.04 \\
85.03,309.10\end{array}$ & \multirow{3}{*}{$\begin{array}{c}\text { feruloyl derivative } \\
\text { cyanidin- } \\
\text { (feruloylhexoside)- } \\
\text { rhamnosylhexoside or } \\
\text { isomer } \\
\text { cyanidin- } \\
\text { (coumaroylhexoside)- } \\
\text { rhamnosylhexoside or } \\
\text { isomer }\end{array}$} \\
\hline 933.2648 & 932.2575 & {$[\mathrm{M}+\mathrm{H}]^{+}$} & 14.5 & 3.0 & $\mathrm{C}_{43} \mathrm{H}_{48} \mathrm{O}_{23}$ & 932.2586 & 1.260 & $\begin{array}{l}177.05,287.05,339.11 \\
321,10,127.04,433.11\end{array}$ & \\
\hline 903.2545 & 902.2472 & {$[\mathrm{M}+\mathrm{H}]^{+}$} & 14.7 & 3.2 & $\mathrm{C}_{42} \mathrm{H}_{47} \mathrm{O}_{22}^{+}$ & 902.2481 & 0.964 & $\begin{array}{c}147.04,287.05,309.10 \\
291.09,433.11\end{array}$ & \\
\hline
\end{tabular}


Table 1. Cont.

\begin{tabular}{|c|c|c|c|c|c|c|c|c|c|}
\hline$m / z$ & $\begin{array}{l}\text { Neutral } \\
\text { Mass }\end{array}$ & Ionization & $\begin{array}{c}\text { RT } \\
\text { (Min) }\end{array}$ & $\log _{2}(\mathrm{FC})$ & $\begin{array}{l}\text { Molecular } \\
\text { Formula }\end{array}$ & $\begin{array}{l}\text { Theoretical } \\
\text { Mass }\end{array}$ & $\begin{array}{c}\text { Mass } \\
\text { Deviation } \\
(\mathrm{ppm})\end{array}$ & Fragments & Tentative Identification \\
\hline 757.2172 & 756.2099 & {$[\mathrm{M}+\mathrm{H}]^{+}$} & 15.0 & 3.2 & $\mathrm{C}_{33} \mathrm{H}_{40} \mathrm{O}_{20}$ & 756.2113 & 1.889 & $\begin{array}{c}287.05,85.03,127.04 \\
433.11\end{array}$ & $\begin{array}{l}\text { cyanidin- } \\
\text { rhamnosylhexoside- } \\
\text { hexoside or } \\
\text { isomer } 2\end{array}$ \\
\hline 369.1184 & 368.1111 & {$[\mathrm{M}+\mathrm{H}]^{+}$} & 15.0 & -1.4 & $\mathrm{C}_{17} \mathrm{H}_{20} \mathrm{O}_{9}$ & 368.1107 & 0.964 & $175.04,207.07,147.04$ & $\begin{array}{l}\text { feruloyl quinic acid isomer } \\
\qquad 1[24]\end{array}$ \\
\hline 611.1599 & 610.1526 & {$[\mathrm{M}+\mathrm{H}]^{+}$} & 15.2 & -1.5 & $\mathrm{C}_{27} \mathrm{H}_{30} \mathrm{O}_{16}$ & 610.1534 & 1.246 & $\begin{array}{c}287.05,85.03,127.04 \\
97.03,145.05\end{array}$ & $\begin{array}{l}\text { cyanidin-dihexoside or } \\
\text { isomer } 2\end{array}$ \\
\hline 394.1136 & 393.1063 & {$[\mathrm{M}+\mathrm{H}]^{+}$} & 15.3 & -1.1 & & & & & \\
\hline 625.1752 & 624.1679 & {$[\mathrm{M}+\mathrm{H}]^{+}$} & 15.5 & 4.0 & $\mathrm{C}_{28} \mathrm{H}_{32} \mathrm{O}_{16}$ & 624.1690 & 1.794 & $\begin{array}{c}317.07,85.03,303.05 \\
127.04,97.03\end{array}$ & $\begin{array}{l}\text { petunidin- } \\
\text { rhamnosylhexoside or } \\
\text { isomer }\end{array}$ \\
\hline 500.1758 & 499.1688 & {$[\mathrm{M}+\mathrm{H}]^{+}$} & 15.8 & 1.6 & $\mathrm{C}_{22} \mathrm{H}_{29} \mathrm{NO}_{12}$ & 499.1690 & 0.459 & $\begin{array}{c}130.07,85.03,160.08 \\
307.10\end{array}$ & indolylacetyl dihexoside \\
\hline 468.1501 & 467.1428 & {$[\mathrm{M}+\mathrm{H}]^{+}$} & 15.8 & 1.6 & & & & & \\
\hline 369.1183 & 368.1110 & {$[\mathrm{M}+\mathrm{H}]^{+}$} & 16.0 & -1.1 & $\mathrm{C}_{17} \mathrm{H}_{20} \mathrm{O}_{9}$ & 368.1107 & 0.774 & $\begin{array}{c}175.04,207.07,147.04, \\
119.05\end{array}$ & $\begin{array}{l}\text { feruloyl quinic acid isomer } \\
\qquad 2[24]\end{array}$ \\
\hline 517.1547 & 516.1474 & {$[\mathrm{M}+\mathrm{H}]^{+}$} & 16.5 & 1.8 & $\mathrm{C}_{22} \mathrm{H}_{28} \mathrm{O}_{14}$ & 516.1479 & 0.903 & $\begin{array}{c}193.05,161.02,85.03 \\
127.04,69.03,97.03\end{array}$ & $\begin{array}{l}\text { caffeoyl quinic acid } \\
\text { hexoside or isomer } 1\end{array}$ \\
\hline 517.1553 & 516.1479 & {$[\mathrm{M}+\mathrm{H}]^{+}$} & 16.8 & 1.5 & $\mathrm{C}_{22} \mathrm{H}_{28} \mathrm{O}_{14}$ & 516.1479 & 0.038 & $\begin{array}{c}193.05,161.02,85.03 \\
127.04,69.03,97.03\end{array}$ & $\begin{array}{l}\text { caffeoyl quinic acid } \\
\text { hexoside or isomer } 2\end{array}$ \\
\hline 393.1893 & 370.1997 & {$[\mathrm{M}+\mathrm{Na}]^{+}$} & 17.6 & 2.1 & $\mathrm{C}_{19} \mathrm{H}_{30} \mathrm{O}_{7}$ & 370.1992 & 1.364 & & \\
\hline 457.2064 & 456.1995 & {$[\mathrm{M}+\mathrm{H}]^{+}$} & 18.8 & 1.9 & $\mathrm{C}_{22} \mathrm{H}_{32} \mathrm{O}_{10}$ & 456.1996 & 0.175 & $\begin{array}{c}\text { 191.14, 147.11, 121.07, } \\
\text { 93.07, 69.07, 209.16 }\end{array}$ & \\
\hline
\end{tabular}

${ }^{1}$ Proposed chemical name based on exact mass and MS/MS fragmentation spectra (please see text and Figure $4 \mathrm{~b}$ for more details). ${ }^{2}$ The retention time of authentic coumaric acid standard was $17.7 \mathrm{~min}$, different from all three detected isomers.

When possible, a molecular formula was assigned to each metabolite based on the exact mass and the isotopic pattern. Tentative identification was performed based on the molecular formula and MS/MS fragmentation pattern. We were able to tentatively assign compound names to 52 out of 67 metabolites. The majority of these compounds are classified as phenolics (80\%). Among them, $70 \%$ are classified as flavonoids (kaempferol, quercetin and anthocyanins derivates, including acyl flavonoids), $18 \%$ as chlorogenic acids (esters of hydroxycinnamic acids and quinic acid), $6 \%$ as hydroxycinnamic acids (ferulic acid and $p$-coumaric acid) and $6 \%$ as coumarins. Flavonoids were identified on the bases of the aglycone fragment (Figure 4a). Deviation of the aglycone $\mathrm{m} / \mathrm{z}$ can be observed in some signals on Table 1 (i.e., 285.04 or 284.03 on kaempferol glycosides) due to homolytic or heterolytic fragmentation. The homolytic fragmentation of flavonoid glycosides produces a radical aglycone ion $\left[\mathrm{Y}_{0}-\mathrm{H}\right]^{-\bullet}(\mathrm{m} / z 284.03$ for kaempferol), whereas the heterolytic fragmentation produces an aglycone fragment ion $\left[\mathrm{Y}_{0}\right]^{-}(\mathrm{m} / \mathrm{z} 285.04$ for kaempferol).

We propose the tentatively identification of the ion at $\mathrm{m} / \mathrm{z} 980.26$ (RT: $11.2 \mathrm{~min}$ ) as kaempferol-sophoroside-(dihydroxymethoxy)-sophoroside (Table 1). The most abundant fragment of this compound corresponds to a kaempferol-sophoroside $(m / z$ 609.15) (Figure $4 \mathrm{~b}$ ). The kaempferol aglycone was also confirmed by the presence of a peak at $m / z$ 284.03. Identification of a kaempferol-3-O-sophoroside-7-O-sophoroside $(\mathrm{m} / \mathrm{z} 934.25)$ was previously reported in B. oleracea [38]. The neutral loss of $m / z 371.11(980.26 \rightarrow 609.15)$ may indicate the loss of an anhydrohexose attached to a dihydroxymethoxy cyclohexane. Finally, the neutral loss of $m / z 47.01(980.26 \rightarrow 933.25)$, supports the hypothesis of the presence of a dihydroxymethoxy group. This is, however, just a proposed structure and a conclusive elucidation will require further analysis.

Significant metabolites are evenly distributed between the groups of compounds with higher and lower concentration in the H-IM population compared to the L-IM population. The only exception is the group of coumarins that showed a higher concentration in the H-IM population. Aside from phenolics, we identified two compounds: kynurenic acid, a product of the kynurenine branch of tryptophan metabolism and an indolylacetyl dihexoside, a carbohydrate derivative. 
(a)



(b)
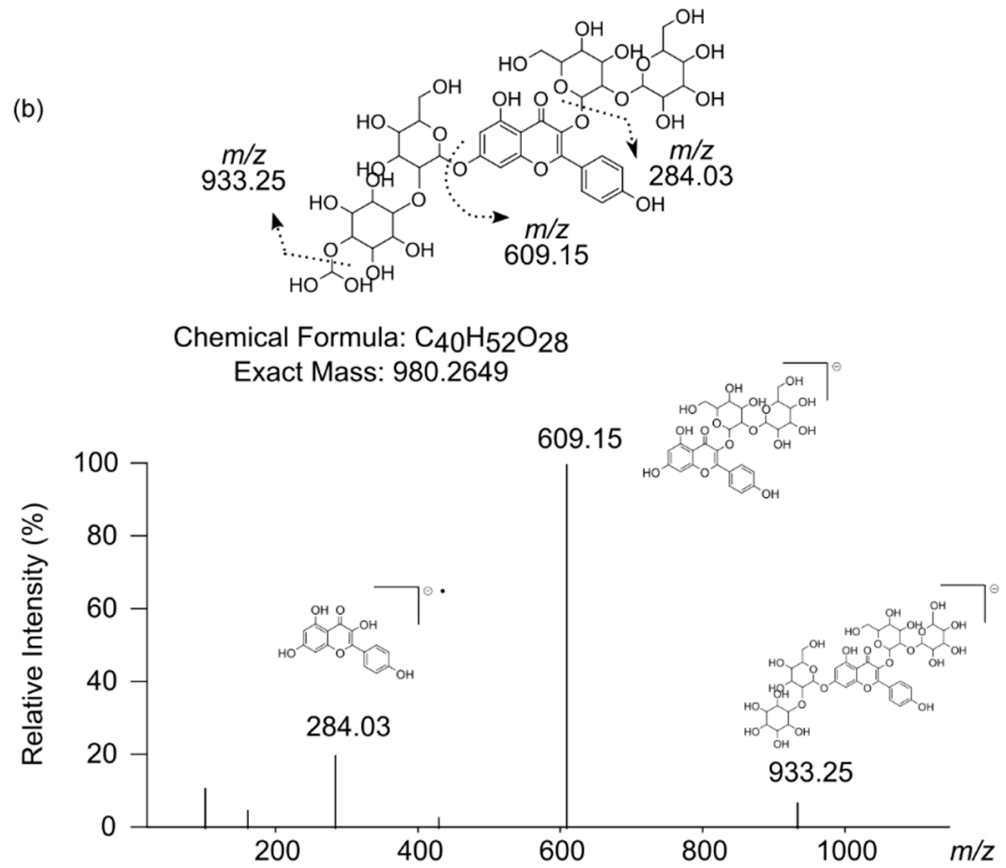

Figure 4. Identification of flavonoids based on MS/MS fragmentation patterns. (a) Ions corresponding to aglycones of the different flavonoids identified in this work. The central structure corresponds to the basic skeleton of flavonoids. The mass of each flavonoid aglycone is reported in brackets. (b) Proposed structure for the $m / z$ 980.2649. Dot lines indicate the hypothetical pattern of fragmentation of the proposed molecule based on the experimental MS/MS mass spectrum obtained.

\section{Discussion}

Changes on the metabolome allow plants to adapt to fluctuations in the environmental conditions. The accumulation of specific metabolites, especially those with antioxidant properties, act as a metabolic buffer under stressful conditions. It has been demonstrated that different environmental and cultivation conditions modify the profile and content of GSLs in Brassica crops [39,40]. In general terms, abiotic stresses tend to increase the content of GSLs in these plants, suggesting that GSLs may play a role on plant adaptation to different environments. However, the direct role of these compounds in plant adaptation 
has yet to be addressed. In this work we used two divergently selection populations (H-IM and L-IM) to study that possible role.

Our results may indicate that the leaf productivity of Brassica crops could be directly or indirectly affected by IM content and that this effect is stable across different environments. We observed a higher yield in the H-IM population in the various experimental locations from southern Spain to northern Norway. To the best of our knowledge this is the first time that the possible role of the IM has been studied directly in relation to local adaptation. Interestingly, both populations barely differ in the amount of other GSLs, so differences in yield could be attributed to a great degree to the accumulation of IM. However, it is plausible that a higher accumulation of IM after three cycles of divergent selection may also produce a reorganization of the plant metabolome, that could contribute to increased plant adaptability.

To study the extent of potential metabolome reorganization we performed an untargeted metabolomics analysis. Extraction conditions $(80 \% \mathrm{MeOH})$, chromatographic setup (reverse-phase UPLC) and ionization interface (ESI) used in our analysis allowed for detection of a wide range of polar and mid-polar metabolites, but with a lack of information about highly polar (elute with the dead volume of the chromatography system) or apolar compounds. With this limitation in mind, our analysis indicate that divergent selection mainly affected phenolic biosynthetic pathways. More than $80 \%$ of the metabolites identified were phenols. Previous studies have reported a simultaneous increase of IM and total phenols in various crucifers (Isatis canescens, B. oleracea var. italica, or B. rapa ssp. rapa) [41-43]. However, our study represents the first example of a possible relationship between higher IM content and higher phenolic compound content in kale.

Phenolic compounds constitute a complex group of secondary metabolites that are widespread in the plant kingdom. They have allelopathic, antimicrobial and antioxidant activity in plants [44-46] and can be precursors of other secondary metabolites (e.g., hydroxycinnamic acids are precursor of lignin) [47]. It is not surprising that most of the phenolics we identified were flavonoids given that they are the most prominent phenolics in Brassica species [24]. Severe stress conditions activate the biosynthesis of flavonoids, which in turn act as an antioxidant system preventing cellular damage. It may be hypothesized that the high levels of IM, a stress-promoted molecule, are perceived by the plant as an indicator of stressful conditions, resulting in the activation of flavonoid biosynthesis. Based on our agronomic results, this is unlikely since plants with an imbalanced defensive response show lower growth rates [48,49]. Some authors suggest a direct or indirect role of flavonoids as growth regulators. Grandmaison et al. [50] reported that flavonoids can regulate cell development by interaction with nuclear proteins. Supporting this idea, Saslowsky et al. [51] demonstrate that the end products of flavonoid biosynthesis are located in the cytoplasm and the nuclei of the tip cells of Arabidopsis roots where they can interact with auxin biosynthesis. In vitro analysis shows that in both subcellular compartments, cytoplasmic and nuclear, flavonoids can also interact with actin, regulating its polymerization [52]. This interaction is structure dependent, with flavonols acting as inhibitors and flavanes as stimulators of actin polymerization [52].

We tentatively identified several derivatives of flavonol (quercetin, kaempferol, (iso) rhamnetin) and anthocyanin (cyanidin, petunidin and delphinidin) that accumulated differentially in the H-IM and L-IM populations. Only cyanidin glycosides, along with the group of coumarins, accumulate in a higher extent in the H-IM than in the L-IM population. Curiously, these two groups of compounds have been reported to inhibit plant development $[53,54]$, so further studies will be necessary to elucidate the role of these compounds in kale growth.

\section{Materials and Methods}

\subsection{Plant Populations}

Two divergently selected kale populations, one with high (H-IM) and one with low (L-IM) IM content, were used in this study. These two populations were selected from a 
local Spanish population (MBG-BRS0062), kept at the Brassica germplasm bank at Misión Biológica de Galicia (MBG-CSIC) (Pontevedra, Spain). These populations had been subjected to three selection cycles (details explained in Sotelo et al.) [21].

\subsection{Growing Conditions and Locations}

Kale seeds (H-IM and L-IM) were sown in multipot-trays in a greenhouse. At the 5-6 leaf stage, plants (50 plants/plot) were transplanted into fields in a randomized block design with two replications. Evaluations were performed during the growing season of 2017 in five locations: Pontevedra (PO) (Spain; $42^{\circ} 26^{\prime} \mathrm{N}, 8^{\circ} 38^{\prime} \mathrm{W}$ ), Badajoz (BA) (Spain; $38^{\circ} 53^{\prime} \mathrm{N}, 6^{\circ} 51^{\prime} \mathrm{W}$ ), Córdoba (CO) (Spain; $37^{\circ} 53^{\prime} \mathrm{N}, 4^{\circ} 42^{\prime} \mathrm{W}$ ), Göttingen (GO) (Germany; $51^{\circ} 32^{\prime} \mathrm{N}, 9^{\circ} 54^{\prime \prime} \mathrm{E}$ ) and Tromsø (TR) (Norway; $69^{\circ} 40^{\prime} \mathrm{N}, 18^{\circ} 56^{\prime} \mathrm{E}$ ). Transplantation and harvest were carried out on 2 April and 26 September (PO), 24 March and 26 September (BA), 5 April and 29 September (CO), 14 June and 31 August (TR) and 16 May and 5 September (GO), respectively. Cultivation operations, fertilization and weed control were carried out according to local practices and crop requirements.

\subsection{Agronomic Parameters}

Fresh weight was quantified using twenty-five fully developed leaves (7th-8th leaf from the apex) from each plot, harvested randomly. The same leaves were subsequently dried at $70^{\circ} \mathrm{C}$ until a constant weight was reached to record the dry mass. Plant height was measured from the soil surface to the base of the upper leaf in 10 plants from each plot.

\subsection{Biochemical Analysis}

For GSLs and non-targeted metabolomics analyses, the 4th leaf from the apex of 15 plants / plot were collected in liquid nitrogen and stored at $-80^{\circ} \mathrm{C}$ until freeze-dried in a lyophilizer (GAMMA 2-16 LSC plus; Christ, Osterode am Harz, Germany). Samples were mechanically milled to a fine powder in a grinder (Janke and Kunkel A10 mill; IKA-LabortechnikStaufen, Staufen, Germany) before metabolite extraction.

\subsubsection{GSLs Analysis}

The analysis of the GSL-profiles in the samples was carried out following the methodology described by [55], with some modifications. Twelve milligrams of freeze-dried kale powder was mixed with $400 \mu \mathrm{L} 70 \%(v / v)$ methanol preheated to $70{ }^{\circ} \mathrm{C}, 10 \mu \mathrm{L}$ of $\mathrm{PbAc}$ $(0.3 \mathrm{M})$ and $120 \mu \mathrm{L}$ of ultra-pure water preheated to $70^{\circ} \mathrm{C}$. Before, $20 \mu \mathrm{L}$ of glucotropaeolin was added as an internal standard. The tubes were shaken in a Microplate incubator (Model OVAN Orbital Midi; OVAN, Badalona, Spain) at $250 \mathrm{rpm}$ for one hour and centrifuged at $3700 \mathrm{rpm}$ for $12 \mathrm{~min}$. Subsequently, $400 \mu \mathrm{L}$ of the glucosinolate extracts was pipetted into an ion-exchange column with Sephadex DEAE-A25 (Sigma-Aldrich, St. Louis, MO, USA). Desulphation was carried out by adding purified sulphatase (E.C. 3.1.6.1, type H-1 from Helix pomatia) (Sigma-Aldrich, St. Louis, MO, USA) solution. Finally, the desulphated GSLs were diluted in $200 \mu \mathrm{L}$ of ultra-pure water and $200 \mu \mathrm{L}$ of $70 \%$ methanol and kept frozen for further analyzes.

The chromatographic analyses were carried out on an Ultra-High-Performance Liquid Chromatograph (UHPLC Nexera LC-30AD; Shimadzu, Kyoto, Japan) equipped with a Nexera SIL-30AC injector (Shimadzu, Kyoto, Japan) and one SPDM20A UV/VIS photodiode array detector (Shimadzu, Kyoto, Japan). The UHPLC column was an X Select ${ }^{\circledR} \mathrm{HSS}$ T3 $(2.5 \mu \mathrm{m}$ particle size, $2.1 \times 100 \mathrm{~mm}$ i.d.) from Waters (Waters Corporation, Milford, MA, USA) protected with a VanGuard pre-column. The oven temperature was set at $35^{\circ} \mathrm{C}$. GSLs were quantified at $229 \mathrm{~nm}$ and were separated by using the following method in aqueous acetonitrile, with a flow of $0.5 \mathrm{~mL} \mathrm{~min}^{-1}$ : $1.5 \mathrm{~min}$ at $100 \% \mathrm{H}_{2} \mathrm{O}$, an 11 min gradient from $5 \%$ to $25 \%(v / v)$ acetonitrile, $1.5 \mathrm{~min}$ at $25 \%(v / v)$ acetonitrile, a minute gradient from $25 \%$ to $0 \%(v / v)$ acetonitrile and a final $3 \mathrm{~min}$ at $100 \% \mathrm{H}_{2} \mathrm{O}$. Specific GSLs were identified by comparing retention times and UV spectra with standards. GSLs standards were purchase from Phytoplan (Diehm \& Neuberger GmbH, Heidelberg, 
Germany). Calibration equations were made with at least five data points, from 0.08 to $1.3 \mathrm{nmol}$ for $3 \mathrm{mSOp}\left(\mathrm{y}=8.16 \times 10^{-6} \mathrm{x} ; \mathrm{R}^{2}=0.99\right)$, from 0.10 to $1.56 \mathrm{nmol}$ for 2-propenyl $\left(y=1.06 \times 10^{-5} \mathrm{x} ; \mathrm{R}^{2}=1.00\right)$, from 0.07 to $1.19 \mathrm{nmol}$ for $\mathrm{IM}\left(\mathrm{y}=3.42 \times 10^{-6} \mathrm{x} ; \mathrm{R}^{2}=1.00\right)$, from 0.07 to $1.09 \mathrm{nmol}$ for $4 \mathrm{MeOIM}\left(\mathrm{y}=2.98 \times 10^{-6} \mathrm{x} ; \mathrm{R}^{2}=0.99\right)$ and from 0.07 to $1.09 \mathrm{nmol}$ for $1 \mathrm{MeOIM}\left(\mathrm{y}=1.5 \times 10^{-6} \mathrm{x} ; \mathrm{R}^{2}=1.00\right)$. The standard curve of IM was used to quantify the amount of $4 \mathrm{HOIM}$ using a response factor of 1 .

\subsubsection{Metabolomic Analysis}

Freeze-dried powder (50 mg) was dissolved in $500 \mathrm{~mL}$ of $80 \%$ aqueous methanol and then sonicated for $15 \mathrm{~min}$. After centrifugation for $10 \mathrm{~min}(16,000 \times g$, at room temperature), the extract was filtered through a $0.20 \mu \mathrm{m}$ micropore PTFE membrane and placed in vials for further analysis. For metabolomic composition analysis we used ultra-performance liquid chromatography coupled with electrospray ionization quadrupole (Thermo Dionex Ultimate 3000 LC; Thermo Fisher Scientific, Waltham, MA, USA) time-of-flight mass spectrometry (UPLC-Q-TOF-MS/MS) (Bruker Compact ${ }^{\mathrm{TM}}$ ) with a heated electrospray ionization (ESI) source. Chromatographic separation was performed in an Intensity Solo 2 C18 column $(2.1 \times 100 \mathrm{~mm} 1.7 \mu \mathrm{m}$ pore size; Bruker Daltonics, Billerica, MA, USA) using a binary gradient solvent mode consisting of $0.1 \%$ formic acid in water (solvent $\mathrm{A}$ ) and acetonitrile (solvent B). The following gradient was used: $3 \% \mathrm{~B}(0-4 \mathrm{~min})$, from 3\% to $25 \% \mathrm{~B}$ (4-16 min), from 25 to $80 \%$ B (16-25min), from 80 to $100 \%$ B (25-30 min), hold 100\% B until $32 \mathrm{~min}$, from $100 \%$ to 3\% B (32-33 min), hold 3\% B until $36 \mathrm{~min}$. The injection volume was $5 \mu \mathrm{L}$, the flow rate was established at $0.4 \mathrm{~mL} / \mathrm{min}$ and column temperature was controlled at $35^{\circ} \mathrm{C}$. MS analysis was operated in spectra acquisition range from 50 to $1200 \mathrm{~m} / \mathrm{z}$. Both polarities $( \pm)$ of ESI mode were used under the following specific conditions: gas flow $9 \mathrm{~L} / \mathrm{min}$, nebulizer pressure $38 \mathrm{psi}$, dry gas $9 \mathrm{~L} / \mathrm{min}$, and dry temperature $220^{\circ} \mathrm{C}$. Capillary and end plate offset were set to 4500 and $500 \mathrm{~V}$, respectively. The instrument was calibrated externally with a calibration solution of $1 \mathrm{mM}$ sodium formate/acetate in $\mathrm{PrOH} / \mathrm{H}_{2} \mathrm{O}$ $50 / 50$ with $0.2 \%$ formic acid directly infused to the source. Before sample injections, LC-qTOF system stability was tested by three consecutive injections of chloramphenicol (ESI-mode; $\Delta \mathrm{RT}=0.02 \mathrm{~min} ; \Delta m / z=0.002)$ and triphenyl phosphate $(\mathrm{ESI}+$ mode; $\Delta \mathrm{RT}=$ $0.02 \mathrm{~min} ; \Delta m / z=0.001)$. The calibration solution was injected at the beginning of each run and all the spectra were calibrated prior to statistical analysis. MS/MS analysis was performed based on the previously determined accurate mass and RT and fragmented by using different collision energy ramps to cover a range from 15 to $50 \mathrm{eV}$. The algorithm T-Rex 3D from the MetaboScape 4.0 software (Bruker Daltonics, Billerica, MA, USA) was used for peak alignment and detection.

\subsubsection{Statistical Analysis}

Parametric statistical analysis was performed using the GLM procedure of SAS 9.4 (SAS Institute Inc., Cary, NC, USA) for agronomic traits and GSLs content. Populations were considered fixed effects and locations were considered random effects. A post hoc ANOVA analysis was performed using the Fisher's protected least significant difference (LSD) at $p \leq 0.05$.

Statistical analysis of metabolomic data was performed using the web-based software Metaboanalyst [56]. In order to remove non-informative variables, data were filtered using the interquantile range filter (IQR). Moreover, Pareto variance scaling was used to remove the offsets and adjust the importance of high- and low-abundance ions to an equal level. The resulting three-dimensional matrix (peak indices, samples and variables) was further subjected to statistical analysis. Univariate analysis (one-way ANOVA) with a $p$ value $\leq 0.05$ was carried out to find differentially expressed metabolites. Using the Volcano Plot (VP) approach, which measure differentially accumulated metabolites based on $t$-statistics and fold changes simultaneously, we also highlighted the metabolites with a $\left|\log _{2}(\mathrm{FC})\right| \geq 1$ and statistically significant difference (FDR $\leq 0.05$ ) between populations. 


\subsubsection{Tentative Metabolite Identification}

Tentative compound identification was performed using accurate mass metabolites reported in different publicly available databases such as METLIN, KEGG, Pubchem, HMDB and Plant Metabolic Network. Additionally, further partial identification of the most significant metabolites was made by comparison of MS/MS fragmentation patterns against reference compounds found in previously mentioned databases and bibliography on plants of the Brassicaceae family.

\section{Conclusions}

We reported a higher yield of H-IM populations of kale across different environments compared to L-IM populations indicating a potentially greater adaptive capacity of the H-IM populations to varied contexts, as measured by a higher production of foliar biomass. The GSL profiles analysis showed a higher content in indole GSLs in H-IM populations, previously described secondary metabolites which are thought to impart higher tolerance to abiotic stresses such as salinity [57] or drought [58]. The H-IM populations of kale had higher concentrations of compounds, which tentatively can be predicted to have antioxidant potential that may contribute to tolerance of abiotic stresses by reducing the generation of reactive oxygen species [59]. The high indole GSL content and the accumulation of other secondary metabolites may give the H-IM populations of kale an improved adaptive capacity under varied environmental conditions, which may be responsible for an observed higher yield of the high indole GSL population.

Supplementary Materials: The following are available online at https:/ /www.mdpi.com/article/ 10.3390/metabo11060384/s1, Table S1: ANOVA analysis of agronomic parameters in two populations of kale from divergently selected populations with varied indol-3-ylmethylGSL (IM) content (H-IM: High IM content; L-IM: Low IM content), Table S2: ANOVA analysis of glucosinolates GSL content in two populations of kale from divergently selected populations with varied in-dol-3-ylmethylGSL (IM) content (H-IM: High IM content; L-IM: Low IM content). 3mSOp (3-(methylsulfinyl)propylGSL), 2-propenylGSL, IM (indol-3-ylmethylGSL), 4HOIM (hydroxyin-dol-3-ylmethylGSL), 4MeOIM (4methoxyindol-3-ylmethylGSL), 1MeOIM (1-methoxyindol-3-ylmethylGSL).

Author Contributions: V.M.R. and P.V. conceived and designed the experiments and performed metabolomics analysis. J.P. analyzed the data and wrote the manuscript. V.M.R., P.V., A.d.H., T.J.J,, A.C.M., C.M., J.A.B.M. and E.O. performed field experiments. All authors have read and agreed to the published version of the manuscript.

Funding: This research and APC was funded by the Ministry of Science and Innovation, of the Government of Spain, grant number RTI2018-096591-B-I00.

Institutional Review Board Statement: Not applicable.

Informed Consent Statement: Not applicable.

Data Availability Statement: The datasets generated during the current study are available from the corresponding author on reasonable request.

Conflicts of Interest: The authors declare no conflict of interest.

\section{References}

1. Francisco, M.; Tortosa, M.; Martínez-Ballesta, M.D.; Velasco, P.; García-Viguera, C.; Moreno, D.A. Nutritional and phytochemical value of Brassica crops from the agri-food perspective. Ann. Appl. Biol. 2017, 170, 273-285. [CrossRef]

2. Šamec, D.; Urlić, B.; Salopek-Sondi, B. Kale (Brassica oleracea var. acephala) as a superfood: Review of the scientific evidence behind the statement. Crit. Rev. Food Sci. Nutr. 2019, 59, 2411-2422.

3. Cartea, M.E.; Velasco, P. Glucosinolates in Brassica foods: Bioavailability in food and significance for human health. Phytochem. Rev. 2008, 7, 213-229. [CrossRef]

4. Poveda, J. Glucosinolates profile of Arabidopsis thaliana modified root colonization of Trichoderma species. Biol. Control 2021, 155, 104522. [CrossRef] 
5. $\quad$ Falk, K.L.; Kästner, J.; Bodenhausen, N.; Schramm, K.; Paetz, C.; Vassão, D.G.; Reichelt, M.; von Knorre, D.; Bergelson, J.; Erb, M.; et al. The role of glucosinolates and the jasmonic acid pathway in resistance of Arabidopsis thaliana against molluscan herbivores. Mol. Ecol. 2014, 23, 1188-1203. [CrossRef] [PubMed]

6. Saladino, F.; Bordin, K.; Luciano, F.B.; Franzón, M.F.; Mañes, J.; Meca, G. Antimicrobial Activity of the Glucosinolates BTGlucosinolates; Mérillon, J.-M., Ramawat, K.G., Eds.; Springer International Publishing: Cham, Switzerland, 2017; pp. 249-274, ISBN 978-3-319-25462-3.

7. Poveda, J.; Eugui, D.; Velasco, P. Natural control of plant pathogens through glucosinolates: An effective strategy against fungi and oomycetes. Phytochem. Rev. 2020, 19, 1045-1059. [CrossRef]

8. Jeschke, V.; Kearney, E.E.; Schramm, K.; Kunert, G.; Shekhov, A.; Gershenzon, J.; Vassão, D.G. How Glucosinolates affect generalist lepidopteran larvae: Growth, development and glucosinolate metabolism. Front. Plant Sci. 2017, 8, 1995. [CrossRef] [PubMed]

9. Knauer, A.C.; Schiestl, F.P. The effect of pollinators and herbivores on selection for floral signals: A case study in Brassica rapa. Evol. Ecol. 2017, 31, 285-304. [CrossRef]

10. Bradburne, R.P.; Mithen, R. Glucosinolate genetics and the attraction of the aphid parasitoid Diaeretiella rapae to Brassica. Proc. Biol. Sci. 2000, 267, 89-95. [CrossRef]

11. Van Loon, J.J.A.; Schoonhoven, L.M. Specialist deterrent chemoreceptors enable Pieris caterpillars to discriminate between chemically different deterrents. Entomol. Exp. Appl. 1999, 91, 29-35. [CrossRef]

12. Jensen, L.; Jepsen, H.; Halkier, B.; Kliebenstein, D.; Burow, M. Natural variation in cross-talk between glucosinolates and onset of flowering in Arabidopsis. Front. Plant Sci. 2015, 6, 697. [CrossRef]

13. Vik, D.; Mitarai, N.; Wulff, N.; Halkier, B.A.; Burow, M. Dynamic modeling of indole glucosinolate hydrolysis and its impact on auxin signaling. Front. Plant Sci. 2018, 9, 550. [CrossRef]

14. Hossain, M.S.; Ye, W.; Hossain, M.A.; Okuma, E.; Uraji, M.; Nakamura, Y.; Mori, I.C.; Murata, Y. Glucosinolate degradation products, isothiocyanates, nitriles, and thiocyanates, induce stomatal closure accompanied by peroxidase-mediated reactive oxygen species production in Arabidopsis thaliana. Biosci. Biotechnol. Biochem. 2013, 77, 977-983. [CrossRef] [PubMed]

15. Martínez-Ballesta, M.C.; Muries, B.; Moreno, D.Á.; Dominguez-Perles, R.; García-Viguera, C.; Carvajal, M. Involvement of a glucosinolate (sinigrin) in the regulation of water transport in Brassica oleracea grown under salt stress. Physiol. Plant 2014, 150, 145-160. [CrossRef]

16. Bonasia, A.; Lazzizera, C.; Elia, A.; Conversa, G. Nutritional, biophysical and physiological characteristics of wild rocket genotypes as affected by soilless cultivation system, salinity level of nutrient solution and growing period. Front. Plant Sci. 2017, 8, 300. [CrossRef] [PubMed]

17. Velasco, P.; Cartea, M.E.; González, C.; Vilar, M.; Ordás, A. Factors affecting the glucosinolate content of kale (Brassica oleracea acephala Group). J. Agric. Food Chem. 2007, 55, 955-962. [CrossRef] [PubMed]

18. Martinez-Ballesta, M.; Moreno-Fernández, D.; Castejon, D.; Ochando, C.; Morandini, P.; Carvajal, M. The impact of the absence of aliphatic glucosinolates on water transport under salt stress in Arabidopsis thaliana. Front. Plant Sci. 2015, 6, 524. [CrossRef]

19. Cartea, M.E.; Velasco, P.; Obregón, S.; Padilla, G.; de Haro, A. Seasonal variation in glucosinolate content in Brassica oleracea crops grown in northwestern Spain. Phytochemistry 2008, 69, 403-410. [CrossRef]

20. Cámara-Martos, F.; Obregón-Cano, S.; Mesa-Plata, O.; Cartea-González, M.E.; de Haro-Bailón, A. Quantification and in vitro bioaccessibility of glucosinolates and trace elements in Brassicaceae leafy vegetables. Food Chem. 2021, 339, 127860. [CrossRef]

21. Sotelo, T.; Velasco, P.; Soengas, P.; Rodríguez, V.M.; Cartea, M.E. Modification of leaf glucosinolate contents in Brassica oleracea by divergent selection and effect on expression of genes controlling glucosinolate pathway. Front. Plant Sci. 2016, 7, 1012. [CrossRef]

22. Santolamazza-Carbone, S.; Velasco, P.; Soengas, P.; Cartea, M.E. Bottom-up and top-down herbivore regulation mediated by glucosinolates in Brassica oleracea var. acephala. Oecologia 2014, 174, 893-907. [CrossRef] [PubMed]

23. Santolamazza-Carbone, S.; Sotelo, T.; Velasco, P.; Cartea, M.E. Antibiotic properties of the glucosinolates of Brassica oleracea var. acephala similarly affect generalist and specialist larvae of two lepidopteran pests. J. Pest Sci. 2016, 89, 195-206.

24. Cartea, M.E.; Francisco, M.; Soengas, P.; Velasco, P. Phenolic compounds in Brassica vegetables. Molecules 2010, 16, 251-280. [CrossRef] [PubMed]

25. Nielsen, J.K.; Olsen, C.E.; Petersen, M.K. Acylated flavonol glycosides from cabbage leaves. Phytochemistry 1993, 34, 539-544. [CrossRef]

26. Wu, S.; Tohge, T.; Cuadros-Inostroza, Á.; Tong, H.; Tenenboim, H.; Kooke, R.; Méret, M.; Keurentjes, J.B.; Nikoloski, Z.; Fernie, A.R.; et al. Mapping the Arabidopsis metabolic landscape by untargeted metabolomics at different environmental conditions. Mol. Plant 2018, 11, 118-134. [CrossRef]

27. Price, K.R.; Casuscelli, F.; Colquhoun, I.J.; Rhodes, M.J.C. Composition and content of flavonol glycosides in broccoli florets (Brassica olearacea) and their fate during cooking. J. Sci. Food Agric. 1998, 77, 468-472. [CrossRef]

28. Yannai, S. Dictionary of Food Compounds with CD-ROM: Additives, Flavors, and Ingredients, 1st ed.; CRC Press: Boca Raton, FL, USA, 2003.

29. Gonzales, G. Mass Spectrometric Characterization of Flavonoids and in vitro Intestinal Transport and Bioactivity Ghent University; Faculty of Bioscience Engineering: Ghent, Belgium, 2016.

30. Llorach, R.; Gil-Izquierdo, A.; Ferreres, F.; Tomás-Barberán, F.A. HPLC-DAD-MS/MS ESI Characterization of unusual highly glycosylated acylated flavonoids from cauliflower (Brassica oleracea L. var. botrytis) agroindustrial byproducts. J. Agric. Food Chem. 2003, 51, 3895-3899. [CrossRef] 
31. Ormrod, D.P.; Landry, L.G.; Conklin, P.L. Short-term UV-B radiation and ozone exposure effects on aromatic secondary metabolite accumulation and shoot growth of flavonoid-deficient Arabidopsis mutants. Physiol. Plant 1995, 93, 602-610. [CrossRef]

32. Olsen, H.; Aaby, K.; Borge, G.I.A. Characterization and quantification of flavonoids and hydroxycinnamic acids in curly kale (Brassica oleracea L. convar. acephala var. sabellica) by HPLC-DAD-ESI-MSn. J. Agric. Food Chem. 2009, 57, $2816-2825$.

33. Rosa, A.S.E. Chemical composition. In Biology of Brassica coenospecies; Gomez-Campo, C., Ed.; Elsevier: Amsterdam, The Netherlands, 1999; pp. 315-357.

34. Martínez-Sánchez, A.; Gil-Izquierdo, A.; Gil, M.I.; Ferreres, F. A Comparative study of flavonoid compounds, vitamin C, and antioxidant properties of baby leaf Brassicaceae species. J. Agric. Food Chem. 2008, 56, 2330-2340. [CrossRef] [PubMed]

35. Tanchev, S.S.; Timberlake, C.F. The anthocyanins of red cabbage (Brassica oleracea). Phytochemistry 1969, 8, 1825-1827. [CrossRef]

36. Wiczkowski, W.; Szawara-Nowak, D.; Topolska, J. Red cabbage anthocyanins: Profile, isolation, identification, and antioxidant activity. Food Res. Int. 2013, 51, 303-309. [CrossRef]

37. Yin, N.-W.; Wang, S.-X.; Jia, L.-D.; Zhu, M.-C.; Yang, J.; Zhou, B.-J.; Yin, J.-M.; Lu, K.; Wang, R.; Li, J.-N.; et al. Identification and characterization of major constituents in different-colored rapeseed petals by UPLC-HESI-MS/MS. J. Agric. Food Chem. 2019, 67, 11053-11065. [CrossRef] [PubMed]

38. Ferreres, F.; Valentão, P.; Llorach, R.; Pinheiro, C.; Cardoso, L.; Pereira, J.A.; Sousa, C.; Seabra, R.M.; Andrade, P.B. Phenolic compounds in external leaves of tronchuda cabbage (Brassica oleracea L. var. costata DC). J. Agric. Food Chem. 2005, 53, 2901-2907. [CrossRef] [PubMed]

39. Biondi, F.; Balducci, F.; Capocasa, F.; Visciglio, M.; Mei, E.; Vagnoni, M.; Mezzetti, B.; Mazzoni, L. Environmental conditions and agronomical factors influencing the levels of phytochemicals in Brassica vegetables responsible for nutritional and sensorial properties. Appl. Sci. 2021, 11, 1927. [CrossRef]

40. Wu, X.; Huang, H.; Childs, H.; Wu, Y.; Yu, L.; Pehrsson, P.R. Glucosinolates in Brassica vegetables: Characterization and factors that influence distribution, content, and intake. Annu. Rev. Food Sci. Technol. 2021, 12, 485-511. [CrossRef]

41. Chung, I.-M.; Rekha, K.; Rajakumar, G.; Thiruvengadam, M. Production of glucosinolates, phenolic compounds and associated gene expression profiles of hairy root cultures in turnip (Brassica rapa ssp. rapa). 3 Biotech. 2016, 6, 175. [CrossRef]

42. Galletti, S.; Barillari, J.; Iori, R.; Venturi, G. Glucobrassicin enhancement in woad (Isatis tinctoria) leaves by chemical and physical treatments. J. Sci. Food Agric. 2006, 86, 1833-1838. [CrossRef]

43. Jo, J.S.; Bhandari, S.R.; Kang, G.H.; Lee, J.G. Comparative analysis of individual glucosinolates, phytochemicals, and antioxidant activities in broccoli breeding lines. Hortic. Environ. Biotechnol. 2016, 57, 392-403. [CrossRef]

44. Fiorentino, A.; Ricci, A.; D’Abrosca, B.; Golino, A.; Izzo, A.; Pascarella, M.T.; Piccolella, S.; Esposito, A. Kaempferol glycosides from Lobularia maritima and their potential role in plant interactions. Chem. Biodivers. 2009, 6, 204-217. [CrossRef]

45. Acquaviva, R.; Russo, A.; Galvano, F.; Galvano, G.; Barcellona, M.L.; Li Volti, G.; Vanella, A. Cyanidin and cyanidin 3-O- $\beta$-Dglucoside as DNA cleavage protectors and antioxidants. Cell Biol. Toxicol. 2003, 19, 243-252. [CrossRef]

46. Razavi, S.M.; Zahri, S.; Zarrini, G.; Nazemiyeh, H.; Mohammadi, S. Biological activity of quercetin-3-O-glucoside, a known plant flavonoid. Russ. J. Bioorganic Chem. 2009, 35, 376-378. [CrossRef] [PubMed]

47. Ferreira, P.S.; Victorelli, F.D.; Fonseca-Santos, B.; Chorilli, M. A review of analytical methods for $p$-coumaric acid in plant-based products, beverages, and biological matrices. Crit. Rev. Anal. Chem. 2019, 49, 21-31. [CrossRef]

48. Rodríguez, V.M.; Chételat, A.; Majcherczyk, P.; Farmer, E.E. Chloroplastic phosphoadenosine phosphosulfate metabolism regulates basal levels of the prohormone jasmonic acid in Arabidopsis leaves. Plant Physiol. 2010, 152, 1335-1345. [CrossRef] [PubMed]

49. Heinrich, M.; Hettenhausen, C.; Lange, T.; Wünsche, H.; Fang, J.; Baldwin, I.T.; Wu, J. High levels of jasmonic acid antagonize the biosynthesis of gibberellins and inhibit the growth of Nicotiana attenuata stems. Plant J. 2013, 73, 591-606. [CrossRef] [PubMed]

50. Grandmaison, J.; Ibrahim, R.K. Evidence for nuclear protein binding of flavonol sulfate esters in Flaveria chloraefolia. J. Plant Physiol. 1996, 147, 653-660. [CrossRef]

51. Saslowsky, D.E.; Warek, U.; Winkel, B.S.J. Nuclear localization of flavonoid enzymes in Arabidopsis. J. Biol. Chem. 2005, 280, 23735-23740. [CrossRef]

52. Böhl, M.; Tietze, S.; Sokoll, A.; Madathil, S.; Pfennig, F.; Apostolakis, J.; Fahmy, K.; Gutzeit, H.O. Flavonoids affect actin functions in cytoplasm and nucleus. Biophys. J. 2007, 93, 2767-2780. [CrossRef]

53. Besseau, S.; Hoffmann, L.; Geoffroy, P.; Lapierre, C.; Pollet, B.; Legrand, M. Flavonoid accumulation in Arabidopsis; repressed in lignin synthesis affects auxin transport and plant growth. Plant Cell 2007, 19, 148-162. [CrossRef]

54. Kupidlowska, E.; Kowalec, M.; Sulkowski, G.; Zobel, A.M. The effect of coumarins on root elongation and ultrastructure of meristematic cell protoplast. Ann. Bot. 1994, 73, 525-530. [CrossRef]

55. Kliebenstein, D.J.; Lambrix, V.M.; Reichelt, M.; Gershenzon, J.; Mitchell-Olds, T. Gene duplication in the diversification of secondary metabolism: Tandem 2-oxoglutarate-dependent dioxygenases control glucosinolate biosynthesis in Arabidopsis. Plant Cell 2001, 13, 681-693. [PubMed]

56. Chong, J.; Wishart, D.S.; Xia, J. Using MetaboAnalyst 4.0 for comprehensive and integrative metabolomics data analysis. Curr. Protoc. Bioinform. 2019, 68, e86. [CrossRef] [PubMed]

57. Steinbrenner, A.D.; Agerbirk, N.; Orians, C.M.; Chew, F.S. Transient abiotic stresses lead to latent defense and reproductive responses over the Brassica rapa life cycle. Chemoecology 2012, 22, 239-250. [CrossRef] 
58. Cavaiuolo, M.; Cocetta, G.; Spadafora, N.D.; Müller, C.T.; Rogers, H.J.; Ferrante, A. Gene expression analysis of rocket salad under pre-harvest and postharvest stresses: A transcriptomic resource for Diplotaxis tenuifolia. PLoS ONE 2017, 12, e0178119. [CrossRef] [PubMed]

59. Hasanuzzaman, M.; Bhuyan, M.H.M.B.; Zulfiqar, F.; Raza, A.; Mohsin, S.M.; Mahmud, J.A.; Fujita, M.; Fotopoulos, V. Reactive oxygen species and antioxidant defense in plants under abiotic stress: Revisiting the crucial role of a universal defense regulator. Antioxidants 2020, 9, 681. [CrossRef] 